\title{
Elektroautos und das Klima: die große Verwirrung
}

\begin{abstract}
Seit einiger Zeit wird erbittert um die Frage gestritten, ob Elektroautos gegenüber konventionellen Fahrzeugen $\mathrm{CO}_{2}$ einsparen oder nicht. Dieser Streit wird allerdings in einer sehr unglücklichen Form ausgetragen. Vor allem basieren die Studien zu diesem Thema auf sehr unterschiedlichen und zum Teil unrealistischen Annahmen. Zudem vernachlässigen sie zwei zentrale Aspekte für die Beurteilung der Elektromobilität sträflich: den Emissionshandel und die Frage der Kosteneffizienz. Im Ergebnis zeigt sich, dass die Förderung von Elektroautos die vermutlich ineffizienteste Form von Klimapolitik ist, die weltweit zurzeit betrieben wird.
\end{abstract}

\begin{abstract}
Viele Politiker sind davon überzeugt, dass eine erfolgreiche Klimapolitik zwei Dinge erfordert: Den Ausbau der erneuerbaren Energien (Energiewende) und den Umstieg des Individualverkehrs auf batterieelektrische Fahrzeuge (BEV), die sogenannte Verkehrswende. Da diese beiden Unterziele gewissermaßen gesetzt sind, gerät bei der klimapolitischen Diskussion das eigentliche Elementarziel, die Reduktion von $\mathrm{CO}_{2}$-Emissionen, häufig aus dem Blickfeld. Dahinter steht die Vorstellung, dass dann, wenn erst alle Verbrennungsmotoren stillstehen, die $\mathrm{CO}_{2}$-Emissionen verschwunden sein werden. Also muss doch das Ziel darin bestehen, alle Motoren auszuschalten, koste es, was es wolle.
\end{abstract}

Eine rationale Klimapolitik müsste jedoch anders vorgehen. Sie müsste anerkennen, dass fossile Brennstoffe nicht kurzfristig komplett verschwinden können und dass es deshalb darauf ankommt, die Reduktion der $\mathrm{CO}_{2}-$ Emissionen möglichst klug zu organisieren. Das bedeutet, dass man dort reduziert, wo die Reduktionskosten am geringsten sind, um auf diese Weise mit den begrenzten

(C) Der/die Autor(en) 2020. Open Access: Dieser Artikel wird unter der Creative Commons Namensnennung 4.0 International Lizenz (https:// creativecommons.org/licenses/by/4.0/deed.de) veröffentlicht.

Open Access wird durch die ZBW - Leibniz-Informationszentrum Wirtschaft gefördert.

Prof. Dr. Joachim Weimann ist Inhaber des Lehrstuhls für Wirtschaftspolitik an der Otto-von-Guericke-Universität in Magdeburg.
Ressourcen, die für die $\mathrm{CO}_{2}$-Vermeidung zur Verfügung stehen, möglichst weit zu kommen. Um eine solche Politik zu organisieren, ist es nicht unerheblich zu wissen, wo und in welchem Umfang Einsparungspotenziale bestehen und wie sie kosteneffizient gehoben werden können. Insofern ist die Frage wichtig, ob durch Elektrofahrzeuge $\mathrm{CO}_{2}$ eingespart werden kann. Die Meinungen darüber gehen weit auseinander.

\section{Studien mit unterschiedlichen Ergebnissen}

Den ersten Stein ins Wasser warfen Buchal et al. (2019). Ihre Analyse zeigte, dass ein BEV der Kompaktklasse gegenüber einem gut konstruierten Diesel-Fahrzeug keine bessere $\mathrm{CO}_{2}$-Bilanz aufweist. Dieses Ergebnis deckt sich mit dem Resultat, zu dem eine Projektion von VW kommt, in der die Einsparpotenziale von BEV im Jahr 2030 geschätzt werden (VW, 2019). Im Unterschied dazu kommen zwei Studien, die aus Instituten stammen, die der Verkehrswende sehr positiv gegenüberstehen, zu gänzlich anderen Ergebnissen. Wietschel et al. (2019) vom Fraunhofer Institut ISI stellen erhebliche Einsparpotenziale fest, und die Agora-Verkehrswende-Studie (2019), die zusammen mit dem Institut für Energie- und Umweltforschung Heidelberg erstellt wurde, bestätigt dies.

Ein Blick in die Studie von Wietschel et al. zeigt schnell, woher die Unterschiede zu beispielsweise Buchal et al. kommen. Ein wichtiger Parameter bei der Berechnung der $\mathrm{CO}_{2}-$ Emissionen von BEV ist die Produktion der Batterie, denn diese ist sehr energieintensiv. Wietschel et al. unterstellen in ihrer Studie für die Mittelklasse eine Batteriegröße von 30 kWh. Ein gängiges E-Auto dieser Klasse (z. B. VW ID.3) verbraucht pro $100 \mathrm{~km} 15 \mathrm{kWh}$, d.h. mit der von Wietschel angenommenen Batteriegröße käme man auf eine maximale Reichweite von $200 \mathrm{~km}$. Deshalb haben E-Autos 
größere Batterien. Der Tesla Modell 3 und der ID.3 haben 75 kWh bzw. 77 kWh Kapazität an Bord. Allein dieser Unterschied macht bei der Batterieproduktion etwa 7 Tonnen $\mathrm{CO}_{2}$-Emission aus.

Wietschel et al. (2019) legen großen Wert auf die Frage, woher der Strom stammt, mit dem Elektroautos betrieben werden. Aufgrund von Befragungsergebnissen stellen sie fest, dass ein großer Anteil derjenigen, die Elektroautos fahren, auch eine Photovoltaik-Anlage besitzen. Sie schließen daraus, dass die Kombination von BEV und Photovoltaik (PV) sehr häufig ist und untersuchen deshalb unter anderem die $\mathrm{CO}_{2}$-Bilanz für den Fall dieser Kombination. Allerdings ist diese Kombination sehr teuer und deshalb vor allem bei Menschen anzutreffen, die über hohe Einkommen verfügen. Wenn es tatsächlich zur Verkehrswende kommt, wird die überwiegende Mehrheit der Konsumenten von BEV nicht in der Lage sein, neben dem teuren E-Auto auch noch eine eigene PV-Anlage zu finanzieren. ${ }^{1}$ Auch die Präsentation der Ergebnisse bei Wietschel et al. wirft einige Fragen auf. So werden die $\mathrm{CO}_{2}-$ Emissionen von BEV in Verbindung mit $100 \%$ PV-Strom und einem Speicher mit etwas unter 5 Tonnen p.a. angegeben (Wietschel et al., 28), obwohl allein die Produktion dieser Fahrzeuge bereits 9 Tonnen (16 Tonnen, wenn man die richtige Batteriegröße unterstellt) $\mathrm{CO}_{2}$-Emissionen verursacht (Wietschel et al., Tabelle 2).

Im Juni 2020 erschien ein kurzer Beitrag von Ulrich Schmidt (2020) als „Policy Brief“-Papier des IfW, in dem darauf hingewiesen wurde, dass bei den Studien der Agora Verkehrswende und des Fraunhofer ISI ein wichtiger Punkt übersehen worden ist. Schmidt macht deutlich, dass der Strom, mit dem ein BEV geladen wird, auch dann nicht $\mathrm{CO}_{2}$-frei ist, wenn er zu $100 \%$ aus einer PV-Anlage kommt. Das ist deshalb der Fall, weil dieser Strom, wenn er nicht dafür genutzt würde, ein Auto anzutreiben, genutzt werden könnte, um fossilen Strom zu ersetzen. Da diese Substitution durch das Elektroauto wegfällt, muss die Emission, die bei dem fossil erzeugten Strom entsteht, dem E-Auto zugerechnet werden. Schmidt geht davon aus, dass deshalb dem BEV eine Emission von $300 \mathrm{~g} / \mathrm{km}$ zuzurechnen ist. Insgesamt würde eine Umstellung auf BEV deshalb dazu führen, dass die $\mathrm{CO}_{2}$-Emissionen um $73 \%$ ansteigen würden.

\section{Berechnung der $\mathrm{CO}_{2}$-Emissionen}

Es ist sehr schwierig, die Studien von Buchal et al. (2019), Wietschel et al. (2019) und Schmidt (2020) zu vergleichen, denn bei der Berechnung der $\mathrm{CO}_{2}$-Emissionen sowohl von BEV als auch von Verbrennern, gibt es eine Reihe

1 Ganz abgesehen davon, dass Deutschland eine Eigenheimquote von unter $50 \%$ hat. von Freiheitsgraden, die unterschiedlich genutzt werden können. Wenn man aus den Studien jeweils die Annahmen heraussucht, die der Realität am nächsten zu liegen scheinen, dann ergibt sich folgendes Bild:

- Die Batteriegröße sollte mit 75 kWh angesetzt werden. Sowohl Buchal et al. als auch Wietschel et al. verwendeten die Meta-Studie von Romare und Dahllöf (2017), um die $\mathrm{CO}_{2}$-Emissionen bei der Batterieproduktion zu schätzen. Diese liegen zwischen $145 \mathrm{~kg} / \mathrm{kWh}$ und $195 \mathrm{~kg} / \mathrm{kWh}$, d.h. sie betragen im Mittel 170 kg/kWh. Grundsätzlich kommt dabei dem Strommix große Bedeutung zu. Viele Studien setzen einfach voraus, dass der Anteil $\mathrm{CO}_{2}$-frei erzeugten Stroms in Zukunft sehr hoch sein wird und rechnen dadurch die durch die Batterieproduktion und den Fahrstrom erzeugten Emissionen klein. Sinnvoller erscheint es aber zu sein, vom Status quo auszugehen und dann zu überlegen, welcher Zuwachs an $\mathrm{CO}_{2}$-freiem Strom notwendig ist, um bestimmte Ziele zu erreichen.

- Laut Wietschel et al. müssen die $\mathrm{CO}_{2}$-Emissionen der Produktion eines Verbrenners bei 6,5 Tonnen angesetzt werden (Mittelklasse). Die eines E-Autos liegen darunter, weil weniger Komponenten produziert werden, deshalb seien sie mit 4 Tonnen angesetzt. Wir unterstellen eine Laufleistung von $15.000 \mathrm{~km}$ p.a. und das zehn Jahre lang. Für das E-Auto ergibt sich dann ein Wert von $111,7 \mathrm{~g} / \mathrm{km} \mathrm{CO}_{2}$-Emissionen, die auf die reine Produktion von Fahrzeug und Batterie zurückzuführen sind. Die Nutzungsdauer eines E-Autos ist eine weitere Stellschraube, an der die Bilanz verändert werden kann. Je länger ein E-Auto auf der Straße ist, umso besser für den Vergleich mit dem Verbrenner. Da bisher keine Erfahrungen mit E-Autos vorliegen, ist es sehr schwer, verlässliche Aussagen zu machen. Ob beispielsweise die Batterie 150.000 km schadlos übersteht, muss letztlich der Praxistest zeigen. Ob E-Autos angesichts der geringen Reichweite auf eine Laufleistung von $15.000 \mathrm{~km}$ pro Jahr kommen, ist ebenfalls sehr fraglich. Die meisten E-Autos dürften die Funktion von Zweitwagen haben, die man für die kurzen Strecken benutzt.

- Unterstellt man den deutschen Energiemix, kommt bei einem Verbrauch von $15 \mathrm{kWh} / 100 \mathrm{~km}$ noch einmal 82,5 g/km Emission durch den Fahrstrom hinzu, sodass das E-Auto insgesamt mit 194,2 $\mathrm{g} \mathrm{CO}_{2} / \mathrm{km}$ unterwegs ist. Unterstellt man, wie Schmidt, dass ausschließlich Braunkohlestrom verwendet wird, ergibt sich eine Fahrstrombelastung von $204 \mathrm{~g} \mathrm{CO}_{2} / \mathrm{km}$, sodass das BEV auf stolze $305,7 \mathrm{~g} \mathrm{CO}_{2} / \mathrm{km} \mathrm{kommt.}^{2}$

2 Dabei wird angenommen, das Braunkohlestrom pro kWh zu einer $\mathrm{CO}_{2}$-Emission von $1369 \mathrm{~g} / \mathrm{kWh}$ führt und der gesamte Strommix zu $550 \mathrm{~g} / \mathrm{kWh}$. Diese Daten sind Buchal et al. (2019) entnommen. 
- Für den Vergleich mit einem Diesel werden die Verbrauchsangaben eines Mittelklassewagens bei Wietschel verwendet $(5,7 \mathrm{l} / 100 \mathrm{~km})$. Buchal et al. rechnen mit $4,5 \mathrm{l} / 100 \mathrm{~km}$, was eine äußerst unrealistische Annahme ist - sie stammt aus einem Werbeprospekt von Mercedes-Benz. Mit Wietschel et al. nehmen wir an, dass pro Liter Diesel einschließlich der Vorproduktion $2,9 \mathrm{~kg} \mathrm{CO}$, emittiert werden. Das entspricht einer Emission von $165 \mathrm{~g} / \mathrm{km}$. Rechnen wir ebenso wie Wietschel mit $\mathrm{CO}_{2}$-Emissionen bei der Produktion des Autos von $6.500 \mathrm{~kg}$, so ergibt sich eine $\mathrm{CO}_{2}$-Emission von $208 \mathrm{~g} /$ $\mathrm{km}$ für das Diesel-Fahrzeug. Nach dieser Rechnung hätte das E-Auto im günstigsten Fall einen $\mathrm{CO}_{2}$-Vorteil von $13,8 \mathrm{~g} / \mathrm{km}$ (entspricht etwa 2 Tonnen über den Lebenszyklus) und im ungünstigsten Fall einen Nachteil von $97,7 \mathrm{~g} / \mathrm{km}$ (entspricht etwa 14,6 Tonnen).

Wie jede andere Rechnung auch, ist diese Berechnung angreifbar, aber sie gibt einen Eindruck von den Größenordnungen und sie zeigt, an welchen Stellen die neuralgischen Punkte liegen. Die Studie von Schmidt (2020) berührt einen solchen Punkt, indem sie darauf aufmerksam macht, dass es unzulässig ist, erneuerbaren Strom mit einer Emission von null anzusetzen. Das ist in der oben angestellten Rechnung insofern geschehen, als die Erneuerbaren in den Strommix mit null Emissionen eingehen. Dass dieser Hinweis von Schmidt ${ }^{3}$ berechtigt ist, steht außer Frage. So kommt auch Wietschel (2020) in einer Stellungnahme zu dem Schmidt-Papier nicht umhin, dieses Argument anzuerkennen. Die Rettung des E-Autos versucht Wietschel in dieser Stellungnahme dadurch zu erreichen, dass er behauptet, dass Braunkohlestrom nicht durch erneuerbare Energien substituiert werden kann ${ }^{4}$ und dass man annehmen müsse, dass Kraftstoffe nur aus Schieferöl in Alaska (!) gewonnen werden, was zu erheblichen Zusatzemissionen führe. Auf diese Weise steigt dann die $\mathrm{CO}_{2}$-Emission der Diesel-Fahrzeuge so stark an, dass das E-Auto wieder im Vorteil ist. Allerdings importiert Deutschland kein Öl aus Alaska.

Auch die Grüne Bundestagsfraktion mochte sich mit den negativen Ergebnissen zum E-Auto nicht abfinden und hat deshalb an der Universität Eindhoven eine Studie in Auftrag gegeben (Hoekstrat und Steinbuch, 2020). Diese Studie bestätigt, dass E-Autos schon jetzt erhebliche $\mathrm{CO}_{2}$-Einsparungen erreichen. Unter den Annahmen, die Hoekstrat und Steinbuch verwenden, beträgt die $\mathrm{CO}_{2}-$ Emission eines E-Autos tatsächlich nur $91 \mathrm{~g} / \mathrm{km}$. Das ist überraschend wenig, und es fragt sich, wie diese gewaltige Differenz zu den anderen Studien zustande kommt.

3 Dieser Hinweis findet sich auch schon bei Buchal et al. (2019).

4 Allerdings behauptet Wietschel im gleichen Beitrag, dass 2050 kein fossiler Strom mehr im Netz sein wird.
Zwei Punkte seien herausgegriffen. Die Produktion der Batterie verursacht in der Studie aus Eindhoven nicht $170 \mathrm{~kg} / \mathrm{kWh}$, sondern nur $87 \mathrm{~kg} / \mathrm{kWh}$. Begründet wird dies damit, dass die Ergebnisse von Romare und Dahllöf (2017) veraltet seien und durch neuere Erkenntnisse von Emilsson und Dahllöft (2019) ersetzt werden müssten. Wenn dies richtig ist, dann hat sich die $\mathrm{CO}_{2}$-Emission bei der Batterieproduktion innerhalb von zwei Jahren halbiert. Das erscheint nicht sehr plausibel, und wenn man sich die Befunde, auf die Hoekstrat und Steinbuch Bezug nehmen, ansieht, dann stellt sich heraus, dass Emilsson und Dahllöft (2019) davon ausgehen, dass die Batterieproduktion fast ausschließlich mit Hilfe von $\mathrm{CO}_{2}$-frei hergestelltem Strom erfolgt. Den gibt es zwar noch nicht (schon gar nicht in China, wo die Produktion überwiegend stattfindet), aber irgendwann wird es soweit sein. Das ist ein Verfahren, das sich bei Studien zu den $\mathrm{CO}_{2}$-Emissionen von elektrischen Antrieben sehr häufig findet. Man unterstellt einfach, dass es irgendwann einmal so viel Strom aus erneuerbaren Energien geben wird, dass elektrische Antriebe weder bei der Produktion noch beim Betrieb nennenswerte Emissionen verursachen. Tatsächlich: Wenn es nur noch Strom aus Erneuerbaren gibt, können elektrische Antriebe gar kein $\mathrm{CO}_{2}$ verursachen. Die Frage ist nur, ob die Annahme gerechtfertigt ist, dass es in überschaubarer Zeit dazu kommt.

Neben der Batterieproduktion wenden Hoekstrat und Steinbuch den Trick mit der zukünftig $\mathrm{CO}_{2}$-freien Stromproduktion auch bei der Berechnung der Emissionen durch den Fahrstrom an. Sie gehen davon aus, dass ein E-Auto in den nächsten 20 Jahren pro Kilowattstunde, die es verbraucht, nur $250 \mathrm{~g} \mathrm{CO}_{2}$ emittiert. Zum Vergleich: Der deutsche Strommix hat gegenwärtig eine $\mathrm{CO}_{2}$-Last von $550 \mathrm{~g} / \mathrm{kWh}$, also mehr als das Doppelte. Um auf durchschnittlich 250 zu kommen, muss man unterstellen, dass die $\mathrm{CO}_{2}$-Last in den nächsten 20 Jahren auf etwa $107 \mathrm{~g} /$ kWh fällt, sich also gegenüber der heutigen Situation um circa $80 \%$ reduziert. Wie das bei stockendem Ausbau der erneuerbaren Energien und der Stillegung der Atomkraftwerke 2022 (immerhin fast $12 \%$ der Stromkapazität) gehen soll, darüber sagen Hoekstrat und Steinbuch leider nichts.

Damit wird klar, dass es entscheidend darauf ankommt, wie schnell die Substitution der fossilen Brennstoffe bei der Stromproduktion gelingt. Das Schmidt-Argument, dass eben nicht mit einer $\mathrm{CO}_{2}$-Emission von null gerechnet werden darf, macht darauf aufmerksam, dass die Substitution umso länger dauern wird, je mehr die Stromnachfrage in der Zukunft ansteigt. Jedes Elektroauto und jede Wärmepumpe, die heute installiert wird, verzögert den Ausstieg aus den fossilen Brennstoffen, weil sie erneuerbaren Strom davon abhält, fossilen Strom zu ersetzen. Aber welchen Effekt hat diese entgangene Substitution? 
An dieser Stelle zeigt sich, dass alle bisher diskutierten Studien die Diskussion nicht weit genug treiben, denn sie übersehen die Rolle, die das europäische Emissionshandelssystem EU-ETS spielt. Der Energiesektor unterliegt dem Emissionshandel, der Verkehrssektor nicht. E-Autos führen dazu, dass der Verkehrssektor quasi in den Energiesektor und damit den Emissionshandel hineinragt. Welche Effekte das hat, ist bisher in der Diskussion vollständig vernachlässigt worden.

\section{Die Rolle des Emissionshandels}

Schmidt (2020) weist im letzten Absatz seines Papiers darauf hin, dass es einen Zusammenhang mit dem Emissionshandel gibt, ohne allerdings wirklich darauf einzugehen. Es zeigt sich schnell, dass die Wirkung des Emissionshandels davon abhängt, in welcher Verfassung das ETS ist. Solange die Obergrenze, der Cap des EU-ETS, bindend ist, d.h. langfristig genauso viele Emissionen getätigt werden, wie Emissionsrechte vergeben wurden, fällt das SchmidtArgument in sich zusammen. In diesem Fall führt die Mehremission von $\mathrm{CO}_{2}$ im deutschen Energiesektor dazu, dass in anderen ETS-Bereichen bzw. in anderen Ländern entsprechend mehr $\mathrm{CO}_{2}$ eingespart wird. Das Aufladen von E-Autos mit Strom aus erneuerbaren Energien führt also nicht zu weiteren Emissionen an anderer Stelle.

Man könnte an dieser Stelle argumentieren, dass mit Hilfe von E-Autos der Verkehrssektor quasi durch die Hintertür in den ETS integriert wird, weil die Produktion des Treibstoffs "Strom" dem ETS unterliegt. Allerdings zeigt sich schnell, dass eine solche Hintertür-Lösung gegenüber einer Vordertür-Lösung, bei der der Verkehrssektor in den ETS integriert wird, deutlich unterlegen ist. Damit ein BEV mit PV-Strom geladen werden kann, muss zunächst einmal eine solche Anlage errichtet werden. Diese Investition, die durch das Erneuerbare-Energien-Gesetz (EEG) subventioniert werden muss, kann man als $\mathrm{CO}_{2}$-Vermeidungs-Investition verstehen. Durch den bindenden Cap ist es notwendig, diese Investition ein zweites Mal zu tätigen, denn sie wird über den Emissionshandel gewissermaßen in den industriellen Sektor des ETS verschoben. Die Vermeidungskosten fallen entsprechend zweimal an. Bei der Vordertür-Lösung würde das entfallen. Integriert man den Verkehrssektor und reduziert die Emissionsrechte, die für diesen Sektor geschaffen werden, nach und nach, kommt es nur dann zum Bau von BEV und PV-Anlagen, wenn dies die kostengünstigste Möglichkeit zur Reduktion von $\mathrm{CO}_{2}$ im gesamten ETS sein sollten. Das kann man ausschließen.

Aber zunächst bleibt noch einmal festzuhalten, dass bei einem funktionierenden Emissionshandel das SchmidtArgument entfällt und es tatsächlich sein kann, dass E-Autos weniger $\mathrm{CO}_{2}$ emittieren als Autos mit Verbrenner. Aller- dings: Wenn der Cap des ETS bindend ist, dann ist auch die Förderung der erneuerbaren Energien in Deutschland komplett redundant. $\mathrm{PV}$ und Windstrom führen dann nicht $\mathrm{zu} \mathrm{CO}_{2}$-Einsparungen, sondern lediglich zu einer kostspieligen Verlagerung der Vermeidung, bzw. der Emission von $\mathrm{CO}_{2}$. Die in Deutschland eingesparten $\mathrm{CO}_{2}$-Mengen werden dann in anderen Ländern des ETS wahrgenommen, weil durch den Einsatz von erneuerbaren Energien die Menge der Emissionsrechte nicht verändert wird. Man spricht in diesem Zusammenhang vom Wasserbetteffekt. Drückt man das Wasserbett an einer Stelle herunter, geht es an anderen Stellen nach oben, die Wassermenge bleibt gleich (Graichen et al., 2018). Unter den Bedingungen eines bindenden Caps ist damit die Förderung erneuerbarer Energien in Deutschland nicht zu rechtfertigen, weil sie sehr teuer ist und keinerlei $\mathrm{CO}_{2}$ einspart. 2018 beliefen sich allein die Zusatzausgaben für die Förderung der erneuerbaren Energien auf 23 Mrd. Euro. Dazu kommen die Kosten des Netzausbaus und -umbaus, die externen Kosten der Windkraftanlagen und vieles mehr. Alles das ist bei einem bindenden Cap verschwendet. Ein bindender Cap wäre deshalb für Agora Verkehrswende eine gute Nachricht, für Agora Energiewende (die sich massiv für die Förderung erneuerbarer Energien einsetzt) aber eine ausgesprochen schlechte.

\section{Folgen der Reform}

2018 wurde der Emissionshandel einer Reform unterzogen, die tiefgreifende Folgen hatte. Ziel der damals eingeführten Marktstabilisierungsreserve war es, den Wasserbetteffekt zu beseitigen. Es ging dabei darum, Reduktionsmaßnahmen, die über den Cap hinausgehen (sogenannte zusätzliche Maßnahmen, wie die Förderung erneuerbarer Energien), vor dem oben beschriebenen Redundanzargument zu schützen. Das Problem: Diesen Effekt kann man nur beseitigen, wenn man die Bindungswirkung des Cap aufhebt - und zwar dauerhaft. Wenn das aber geschieht, dann setzt man damit den Emissionshandel außer Kraft, weil dann der Preis für die Emissionsrechte auf null fallen wird (Weimann und Timme, 2019). Ohne Wasserbetteffekt verlieren wir den Emissionshandel und damit das wirksamste und kosteneffizienteste Klimaschutzinstrument, das sich weltweit gegenwärtig finden lässt. Das wäre ein sehr hoher Preis dafür, die Förderung der erneuerbaren Energien in Deutschland weiter politisch rechtfertigen zu können. ${ }^{5}$

5 Dieser Umstand wird bisher in der Literatur nicht gesehen. So plädiert auch der Sachverständigenrat zur Begutachtung der gesamtwirtschaftlichen Entwicklung dafür, den Wasserbetteffekt zu beseitigen (Sachverständigenrat, 2019). 
Die Reform 2018 hat dafür gesorgt, dass die Bindungswirkung des Cap - und damit der Wasserbetteffekt - vorübergehend $^{6}$ aufgehoben wird. Das geschieht dadurch, dass überschüssige Emissionsrechte in eine Reserve eingestellt werden, aus der heraus ab 2023 die Rechte, die über eine Höchstgrenze hinausgehen, gestrichen werden. Zumindest bis 2025 verschwindet damit die Redundanz des EEG. Wird bis dahin durch den Einsatz erneuerbarer Energien in Deutschland $\mathrm{CO}_{2}$ eingespart, führt das dazu, dass entsprechend mehr Rechte gelöscht werden können. Dieses Mal ist das für Agora Energiewende eine gute Nachricht. Für Agora Verkehrswende aber eine sehr schlechte. Denn damit gilt das Schmidt-Argument wieder in vollem Umfang: Wird jetzt ein E-Auto mit erneuerbarem Strom geladen, wird die Reserve des ETS kleiner, und es werden weniger Rechte gelöscht und damit weniger $\mathrm{CO}_{2}$ eingespart.

Man sieht, dass die Dinge einigermaßen kompliziert werden, wenn man alle klimapolitischen Regulierungen berücksichtigt. Das ist deswegen so, weil die Regulierungsdichte so hoch ist und offensichtlich die politisch Verantwortlichen und die innen zuarbeitenden Institute längst den Überblick verloren haben. Anstatt den Ausbau der Erneuerbaren zu fördern, das EU-ETS ständig $z u$ verschlimmbessern und E-Autos zu subventionieren, wäre es viel einfacher, effizienter und klimaschonender, den Verkehrssektor in das ETS zu integrieren. Das könnte ein Schlusswort sein, aber die Geschichte ist leider noch nicht zu Ende.

\section{Die Frage der Kosteneffizienz}

Vieles spricht dafür, dass E-Autos im Vergleich zu Verbrenner Fahrzeugen kaum $\mathrm{CO}_{2}$ vermeiden. Aber selbst wenn sie das täten, sollte man sie deshalb fördern? Mit den Worten des Sachverständigenrats für Wirtschaft ist eine $\mathrm{CO}_{2}$-Vermeidung effizient, wenn „die jeweils nächste Einheit Treibhausgasemission dort eingespart wird, wo dies nach dem aktuellen Stand der (technischen) Gegebenheiten am günstigsten möglich ist." (Sachverständigenrat, 2019, Ziffer 7).

Stellt sich die Frage, ob die Elektromobilität als technische Gegebenheit dafür infrage kommt, die günstigste Einsparmöglichkeit zu liefern. Um diese Frage zu beantworten, muss man eine ungefähre Vorstellung davon haben, wie hoch die Einsparungen bestenfalls sein könnten. Wenn wir die oben angestellte Rechnung zugrunde legen, den Schmidt-Effekt vernachlässigen und von einem bin-

6 Langfristig ist der Cap nach wie vor bindend, deshalb ist der Preis bisher nicht auf null gefallen. Er ist sogar gestiegen, weil er durch die Streichung von Rechten früher bindend wird und die Verknappung dann zu einem Preisanstieg führen wird, der sich schon an den Börsen zeigt, weil bereits heute Rechte für die Zukunft erworben werden. denden Cap im ETS ausgehen, ${ }^{7}$ dann ist eine Größenordnung von weniger als 10 Tonnen realistisch. Gehen wir, um gewissermaßen auf der sicheren Seite zu sein, von 10 Tonnen Einsparung aus. Was kostet diese $\mathrm{CO}_{2}$-Ersparnis? Auch dafür sei hier nur ein grober Orientierungswert angenommen. Wir benutzen die Subventionen und Kostenvorteile, die bei einem E-Auto dazu führen, dass es ähnliche Kosten aufweist wie ein Verbrenner, als groben Anhaltspunkt für den Kostenunterschied.

Gegenwärtig wird der Kauf eine E-Autos mit insgesamt 9.000 Euro subventioniert (6.000 Euro davon trägt der Staat, 3.000 Euro die Industrie). Dazu kommen der Verzicht auf die Kfz-Steuer für zehn Jahre, der überschlägig noch einmal mit 2.000 Euro bis 3.000 Euro zu Buche schlägt, und weitere Steuersubventionen. ${ }^{8}$ Diese Subventionen erfolgen, um den Kostenunterschied zu Verbrenner-Fahrzeugen auszugleichen. Lassen wir es dabei und verzichten darauf, die Kosten zu berücksichtigen, die durch die reduzierten Reichweiten und die langen Ladezeiten in Form von Opportunitätskosten entstehen. Die genannten Subventionen führen zusammen mit dem Wartungskostenvorteil von E-Fahrzeugen dazu, dass für den Endkunden das E-Auto etwa genauso teuer ist wie ein Diesel-Fahrzeug. Das lässt die Vermutung zu, dass der Kostenunterschied in der Größenordnung von 11.000 Euro bis 12.000 Euro liegen dürfte (Untergrenze). Bei 10 Tonnen $\mathrm{CO}_{2}$-Einsparung bedeutet das Vermeidungskosten von 1.100 Euro bis 1.200 Euro/ Tonne. Nicht berücksichtigt sind die Kosten für den Aufbau einer E-Auto-Infrastruktur, der Verlust von einigen zehntausend Arbeitsplätzen in der Automobilbranche und die bereits angesprochenen Opportunitätskosten. Angesichts der jüngsten Fortschritte bei der Abgasreinigung von Verbrenner-Motoren fallen Einsparungen bei anderen Schadstoffen kaum mehr ins Gewicht.

Gegenwärtig liegen der Grenzvermeidungskosten im ETS-Sektor bei etwa 25 Euro/Tonne. Das bedeutet, dass die Vermeidungskosten beim E-Auto um den Faktor 44 bis 48 höher liegen. Selbst wenn wir eine Vervierfachung des $\mathrm{CO}_{2}$-Preises unterstellen, dürfte ein E-Auto nur 1.000 Euro mehr kosten als ein Verbrenner, um eine effiziente Alternative zu sein. E-Autos erreichen nicht einmal die Nähe eines Verdachts, dass sie in absehbarer Zeit die kostengünstigsten Vermeidungsalternative darstellen können. Man kann

7 Würde man als Vergleichsfahrzeuge Voll-Hybrid-Antriebe heranziehen, würde die Bilanz noch einmal deutlich schlechter für die E-Autos ausfallen, denn dieser Antrieb ist noch einmal deutlich effizienter als ein konventioneller Diesel. Dazu kommt, dass bei dieser Technik kein Strom zugeführt wird, es also nicht zu dem Schmidt-Effekt kommt und der Aufbau einer Ladeinfrastruktur entfällt. Übrigens werden VollHybrid-Fahrzeuge von der Bundesregierung nicht gefördert!

8 Beispielsweise ist Strom, der zum Laden eines BEV vom Arbeitgeber überlassen wird, nicht als geldwerter Vorteil zu versteuern. 
es auch anders ausdrücken: Es gibt vermutlich kaum eine andere Form der $\mathrm{CO}_{2}$-Vermeidung, die noch teurer, noch verschwenderischer und noch ineffizienter ist als der Bau und der Betrieb von BEV - und das unter der Annahme, dass der Schmidt-Effekt keine Rolle spielt.

Würden wir uns auf die Frage der Kosteneffizienz konzentrieren, dann erübrigen sich die mitunter kleinlichen Debatten um die Frage, wie viel $\mathrm{CO}_{2}$ ein E-Auto nun einspart. Angesichts der gegenwärtig horrenden Kosten wäre des E-Auto auch dann keine effiziente Alternative, wenn es gar keine Emissionen verursachen würde. Da nicht in Aussicht steht, dass die Kosten in einigermaßen absehbarer Zeit drastisch fallen, wird sich an dieser Einschätzung auch so schnell nichts ändern können. Die Schlussfolgerung ist, dass wir keine „Verkehrswende“ brauchen, die mit der Brechstange E-Autos in den Markt hebelt, sondern eine konsequente Integration des Verkehrssektors in den ETS.

Die Bundesregierung hat in ihrem Klimapaket von 2019 beschlossen, die E-Mobilität weiter massiv zu fördern. Obwohl eine Integration des deutschen Verkehrssektors in den EU-ETS möglich gewesen wäre, hat sie darauf verzichtet. Außerdem hat die Bundesregierung 2020 im Zuge des Konjunkturpakets, das den Folgen der Corona-Pandemie entgegenwirken soll, beschlossen, die Förderung der E-Mobilität noch einmal zu erhöhen. Beide Entscheidungen müssen vor dem Hintergrund der hier angestellten Überlegungen als klimapolitisch sehr problematisch eingeschätzt werden.

\section{Literatur}

Agora Verkehrswende (2019), Klimabilanz von Elektroautos. Einflussfaktoren und Verbesserungspotenzial, https://www.agora-verkehrswen-
de.de/fileadmin/Projekte/2018/Klimabilanz_von_Elektroautos/AgoraVerkehrswende_22_Klimabilanz-von-Elektroautos_WEB.pdf (28. Juli 2020).

Buchal, C., H.-D. Karl und H.-W. Sinn (2019), Kohlemotoren, Windmotoren und Dieselmotoren: Was zeigt die $\mathrm{CO}_{2}$-Bilanz?, ifo Schnelldienst, 72(08), 40-54.

Emilsson, E. und L. Dahllöf (2019), Status 2019 on Energy Use, CO Emissions, Use of Metals, Products Environmental Footprint, and Recycling, Diskussionspapier, C 444, IVL Swedish Environmental Research Institute.

Graichen, P., F. Matthes, P. Litz und H. Hermann (2018), Vom Wasserbett zur Badewanne, Die Auswirkungen der EU-Emissionshandelsreform 2018 auf $\mathrm{CO}_{2}$-Preis, Kohleausstieg und den Ausbau der Erneuerbaren, Agora Energiewende und Öko-Institut.

Hoekstra, A. und M. Steinbuch (2020), Comparing the lifetime green house gas emissions of electric cars with the emissions of cars using gasoline or diesel, Universität Elndhover, https://www.oliver-krischer. eu/wp-content/uploads/2020/08/English_Studie.pdf (17. September 2020).

Romare, M. und L. Dahllöf (2017), The Life Cycle Energy Consumption and Greenhouse Gas emissions from Lithium-Ion Batteries: A Study with Focus on Current Technology and Batteries for Light-duty Vehicles, Swedish Environmental Research Institute.

Sachverständigenrat (Sachverständigenrat zur Begutachtung der gesamtwirtschaftliche Entwicklung), Sondergutachten (2019), Aufbruch zu einer neuen Klimapolitik, https://www.sachverstaendigenrat-wirtschaft.de/fileadmin/dateiablage/gutachten/sg2019/sg_2019.pdf (13. November 2020).

Schmidt, U. (2020), Elektromobilität und Klimaschutz: Die große Fehlkalkulation, IWF Policy Brief, Nr. 143.

VW (2019), Klimabilanz von E-Fahrzeugen \& Life Cycle Engineering, https://uploads.volkswagen-newsroom.com/system/production/ uploaded_files/14448/file/da01b16ac9b580a3c8bc190ea2af27db4e0d4546/Klimabilanz_von_E-Fahrzeugen_Life_Cycle_Engineering.pdf?1556110703 (16. Juni 2019).

Weimann J. und F. Timme (2019), Die Entfremdung des Emissionshandels, Energiewirtschaftliche Tagesfragen, 69(5).

Wietschel, M. (2020), Stellungnahme zum Policy Brief Elektromobilität und Klimaschutz: Die große Fehlkalkulation, Fraunhofer-Institut für System- und Innovationsforschung ISI, 22. Juni, https://www.isi. fraunhofer.de/content/dam/isi/dokumente/cce/2020/Stellungnahme_ IfW-Langfassung.pdf (28. Juli 2020).

Wietschel, M., M. Kühnbach und D. Rüdiger (2019), Die aktuelle Treibhausgasemissionsbilanz von Elektrofahrzeugen in Deutschland, Fraunhofer ISI Working Paper Sustainability and Innovation, Nr. S 02/2019.

Title: Electric Cars and the Climate: The Big Confusion

Abstract: The discussion about electro mobility revolves primarily around the question of whether electric cars (BEV) emit less $\mathrm{CO}_{2}$ than modern internal combustion vehicles. This demonstrates that there are many degrees of freedom in the comparison, which favor either one side or the other, making the situation very confusing. Weighty aspects in the discussion are either not considered at all or only marginally. For example, the fact that substituting BEVs for combustors would mean that the replacement of coal-fired power would proceed more slowly. Furthermore, the influence of emissions trading is not considered and the question of cost efficiency is not addressed.

JEL Classification: D62, Q42, Q52, Q58 